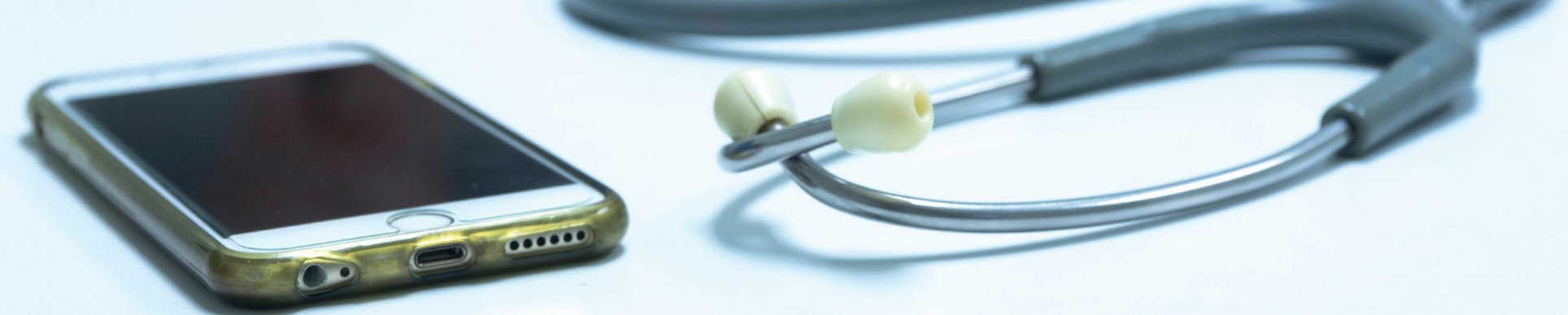

\title{
OUT OF THE BOX:
}

DIAGNOSING CONGUSSIONS WITH A SMARTPHONE

Two Purdue University students founded brightlamp, LLC, a company designed to produce consumer-ready software for medical diagnostics, which is currently focused on immediate concussion detection.

Concussions appear prevalent in the athletic industry today. Although safety for athletes should be the number one priority, concussive injuries occur, as demonstrated through countless lawsuits against the NFL. The existing safety precautions in today's athletic industry do not take advantage of the technology that is currently available.

Brightlamp, LLC is a computational medicine company that produces consumer-ready software for medical diagnostics. The company's mission is make the world medically mobile by reforming the way we interact with technology and our health. The goal is to bring health care into the hands of everyday people by allowing them access to simple diagnostic services for a fraction of the cost. One of the most difficult challenges in today's world is the lack of understanding regarding the potential of new innovations. Often advanced technology is thwarted by its application. Specifically, the medical sector has failed to keep medicine up to speed with current technologies. Brightlamp exists as the bridge to place medicine where it is needed most: in patients' phones.

Kurtis Sluss and Jonathan Holt founded the company in November 2015. Both of the individuals were undergraduates at the time and were competing in a startup competition. Sluss is a machine learning engineer who first discovered this field at Purdue when he was classifying cancerous proteins to determine whether or not an individual had breast cancer. With their backgrounds in engineering and a passion for innovation, the two formulated a business centered around the idea of a more medically mobile world. The company's invented algorithm utilizes a statistical computer vision method and a neural network to classify head trauma in individuals. In collaboration with Dr. Eric Nauman, the team was able to establish partnerships with local high schools and universities to help further validate their technology.

Brightlamp's introductory diagnostic product is a mobile application that aims to detect head trauma. The company's innovative method only requires a camera and flash to measure the extent of the injury, which only takes 5 seconds. The application will begin testing in late 2018 with a full release later that year. With the help of computer models and synthetic data, the application has shown accuracy greater than $98 \%$. With the help of a neural network, brightlamp's algorithm will take data in and update the detection method to continuously deliver the best accuracy on the market.

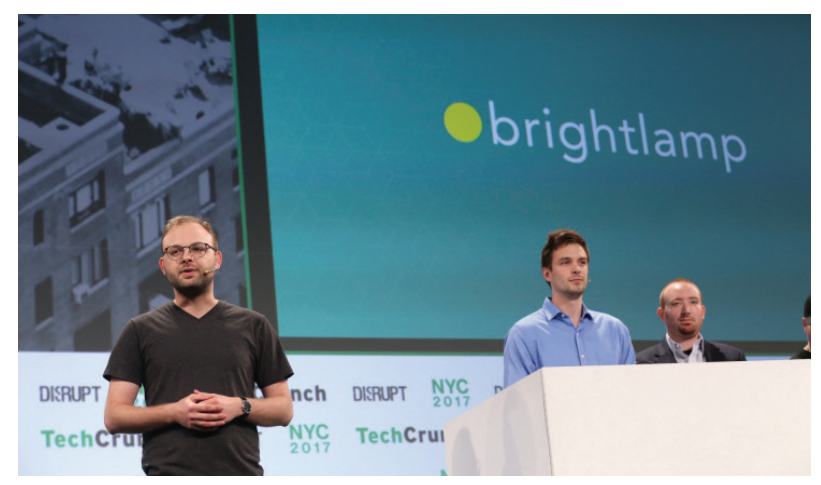

Brightlamp LLC's introductory mobile application aims to detect head trauma by analyzing the pupil using only the camera, flash, and processor found in a smartphone. 
An estimated $84 \%$ of all concussions go undiagnosed. With this astounding number, it is clear that insufficient test methods to detect head trauma are being used. Many applications are available for athletic departments, including Sway Balance, ImPACT surveys, eye tracking, and so forth. Despite these methods and more, there is an epidemic plaguing athletes. With the implementation of brightlamp's concussion detection application, not only do athletic departments and hospitals have access to stateof-the-art technology, but private consumers will have the ability to test themselves and loved ones. No concussion diagnostic application exists on the market today that can deliver such high accuracy and quick results directly to a personal mobile phone.

\section{"Healthcare should be accessible and affordable for all."}

\section{Kurtis Sluss, CEO of brightlamp, LLC}

Recently, the team competed at Tech Crunch Disrupt in New York as one of the battlefield teams. Out of more than one thousand applications, brightlamp was one of seventeen teams to be selected to present at the conference. Brightlamp's innovative method to detect concussions on a mobile device is now patent pending. Application of this technology to a mobile suite is still in development. This technology promises many more diagnostic methods that are currently being investigated and integrated into the platform. After the launch of the pilot application for concussion detection, the team plans to continually release mobile application updates that will target the medical industry to distribute affordable health care to the general consumer. Making the world medically mobile is brightlamp's mission, one application at a time.

Sluss, K., Holt, J., Wilhite, C., Heims, M., Waggoner, J., \& Sinks, D. (2017). Out of the box: Diagnosing concussions with a smartphone. Journal of Purdue Undergraduate Research, 7, 86-88. https://doi. org/10.5703/1288284316415
Student Authors

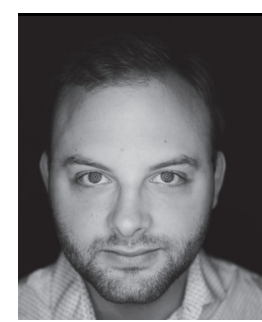

Kurtis Sluss is a 2016 Purdue graduate in chemical engineering and chemistry. He is an engineer, inventor, and entrepreneur. Migration of current diagnostic methods onto a mobile device requires a mindset between frontier programming and advanced medicine. Sluss exercises this mindset to help the brightlamp team formulate new disruptive technologies in the most effective and economical way possible. He leads brightlamp as cofounder and $\mathrm{CEO} /$ president with experience in innovative detection and diagnostic methods and an intuition for startup success.

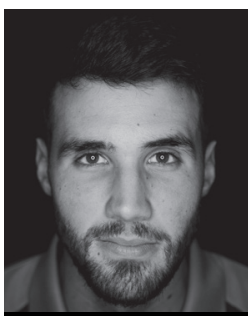

Jonathan Holt is a 2017 Purdue graduate in electrical engineering. His background in engineering has helped develop critical and strategic plans to help shape and grow the company. He is dedicated to intellectual property acquisition and focuses on advancing brightlamp's product protection. He leads the team in patent, trademark, and copyright law while serving as cofounder and vice president. In collaboration with the Indiana University Law Clinic, Holt has secured all intellectual property rights regarding the current implementation of the application on smartphones.

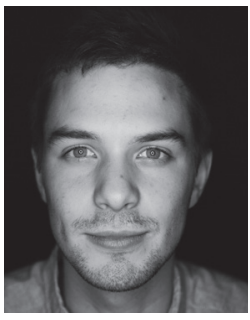

Craig Wilhite is a 2017 Purdue graduate in computer science. He has pioneered brightlamp's vision to bring medical diagnostics to a mobile platform. His revolutionary development with the company's concussion platform as chief technology officer is unprecedented, and he aims to make the world safer through software.

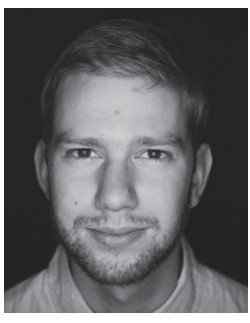

Michael Heims is a 2016 Purdue graduate in management, finance, and supply chain. He has served brightlamp as the chief financial offer, with expertise in the medical sector. He was instrumental in developing the company's valuation, revenue, and business models.

Heims continues his work in the medical sector while offering support to brightlamp by all appropriate means. 


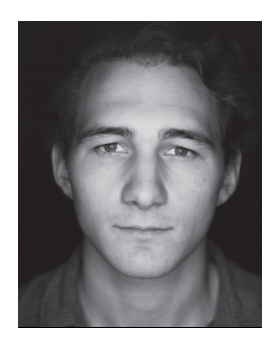

James Waggoner will be a 2018 Purdue graduate in mechanical engineering. His methods in videography and photography are on the frontier of modern techniques. As the chief marketing officer, he demonstrates brightlamp's dramatic vision and impact.

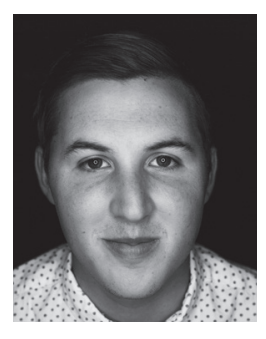

Dylan Sinks is a 2016 Purdue graduate in biomedical engineering. $\mathrm{He}$ focuses on quality and regulatory affairs. As the chief compliance officer, he ensures brightlamp complies with internal policies, is on track with a successful regulatory pathway, and puts forth a quality product.

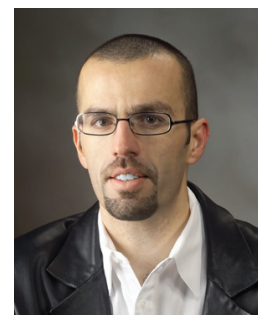

Pavlos Vlachos is a full professor in mechanical engineering at Purdue University and serves as the director of device development at brightlamp. Vlachos conceived the statistical computer vision algorithm that is implemented in the dynamic pupilometer and diagnostic head trauma application. Beyond the initial algorithm, Vlachos is conceptualizing new applications that use the same pupillometry scope as a backbone.

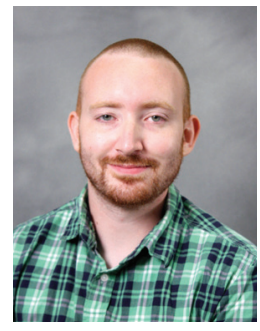

Brett Meyers is a PhD candidate in mechanical engineering at Purdue University and serves as the director of applied vision systems with brightlamp. His research focuses on design and implementation of statistical computer vision algorithms in biomedical flows.

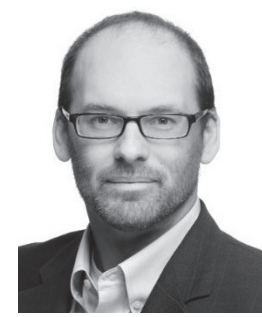

Michael Shepard serves as entrepreneur-in-residence for Elevate Ventures at the Purdue Foundry, where his primary responsibilities include providing guidance to entrepreneurs on business plans, prototype development, funding, grant writing, regulatory requirements, mentoring, and other entrepreneurial activities. Shepard was assigned to brightlamp's team at their infancy of development and has since offered numerous review sessions and advice to continue rapid growth.

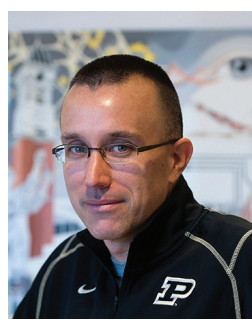

Eric Nauman is brightlamp's technology and research adviser. He currently is an award-winning professor at Purdue University with a research focus on mild traumatic brain injury. Nauman is developing new test methods for the company's technology with his extensive knowledge in sports-related head trauma and a research group dedicated to its long-term effects on the brain. Additionally, he offers supplemental advice on acquiring FDA certification and works closely with grant writers to acquire additional capital to fund brightlamp's research. 